\title{
Numerical characterization of a technically premixed hydrogen flame under conditions close to flashback
}

\author{
D. Mira ${ }^{1}$, O. Lehmkuhl ${ }^{1}$, A. Both ${ }^{1}$ P. Stathopoulos ${ }^{2}$, T. \\ Tanneberger $^{2}$, T. G. Reichel ${ }^{2}$, C. O. Paschereit ${ }^{2}, M$. \\ Vázquez $^{1}$, G. Houzeaux ${ }^{1}$ \\ 1 CASE Department, Barcelona \\ Supercomputing Centre, Barcelona, Spain \\ 2 Hermann-Föttinger-Institut, Technische Universität \\ Berlin, Berlin, Germany
}

Received: date / Accepted: date

\begin{abstract}
This work presents a numerical study of a technically premixed swirling combustor with central air injection at conditions close to flashback using large-eddy simulation with flamelet modelling. The study shows the assumption of perfect premixing is valid during the stable operation of the burner up to flashback conditions. The experimental results are well predicted under inert and reacting conditions by using a perfectly premixed mixture. It is found that the non reacting flow field develops a self-excited oscillation in the form of a precessing vortex core. This oscillation is attenuated by the fuel injection due to the respective increase in axial momentum and it is ultimately suppressed in the reacting flow field. Both experiments and simulations confirm the same trends. The analysis of the flames have shown certain dynamics as the flashback point is approached. The flashback resistance of the burner is minimized due to an increase in the velocity deficit of the incoming mixture. The recirculation region is shifted upstream, the central recirculation is altered and the flame position is displaced towards the reactants. OH-PLIF measurements are compared with the $\mathrm{OH}$ predictions by the LES and certain level of disagreement is observed. This modelling approach is found to be valid to predict the hydrodynamic behaviour of the flames in terms of velocity fields and flow oscillations, but it can not predict the $\mathrm{OH}$ formation found in the post combustion zone across the reacting layer.
\end{abstract}

Keywords premixed burner $\cdot$ swirl-stabilized flames $\cdot$ flashback safety $\cdot$ precessing vortex core · flamelet

\section{Introduction}

The use of swirling combustors is widely spread in modern gas turbines because of the high enhancement of mixing and the resulting compact and low emissions flames [29]. The axial pressure gradient in the swirling flow generates a vortex breakdown with a central recirculation zone, which acts as a flame holding mechanism. These flames are well-known to be

Address(es) of author(s) should be given

This is a post-peer-review, pre-copyedit version of an article published in Flow, turbulence and combustion.

The final authenticated version is available online at: http://dx.doi.org/10.1007/s10494-019-00106-z 
stable for a wide range of operating conditions, due to the interaction of the reversed flow with the high turbulence in the shear layers that increase the turbulent burning velocity [7]. Swirl-stabilized flames are commonly used in premixed burners to operate under lean conditions in order to reduce $\mathrm{NO}_{x}$, but are not exempt of technological challenges [15],27]. Some of the major issues include thermo-acoustic instabilities due to the interaction of pressure fluctuations and heat release [30], self-excited flow oscillations that result in a precessing vortex core (PVC) with large-scale helical flow structures [22] or lean-blow out (LBO) [38], which ultimately contribute to reducing the flexibility of operation under lean conditions. In general, the PVC is synchronized with helical Kelvin-Helmholtz instabilities that tend to trap the fuel within the PVC leading to strong temporal fuel concentration fluctuations [8]. There has been many efforts dedicated to the study and the suppression of this instability [3, 13, 14,22]. Several mechanisms can be identified as responsible for the damping in both isothermal and reacting flows. Those include a reduction of the tangential velocities near the jet centerline [29], flame-induced dilatation and increased viscosity [26] or density stratification [22].

An important parameter in premixed combustion is the flashback resistance of the burner. This is particularly important when combustors are operated with alternative fuels with varying composition or at part load [38]. In this context, swirling combustors without a bluffbody can take advantage of an axial air injection that reduces the velocity deficit along the centerline, thus providing additional resistance against flashback [25]24]. Axial air injection influences the location of the flame by displacing the stagnation point of the aerodynamically induced recirculation downstream in the combustion chamber, thus increasing burner stability. Burners operated with hydrogen-rich fuels, usually require high volumetric flow rates as hydrogen is very light. This introduces a significant amount of additional momentum that can be used for flashback safety. An example of this design is the technicallypremixed swirl-stabilized burner developed in the framework of the European Union FP7 project Advanced Hybrid Engines for Aircraft Development (AHEAD). Experimental measurements [24] show that flashback appears at low equivalence ratios, when this combustor is operated with pure hydrogen. This seems to be a contradictory result, since leaner mixtures become less reactive and should be less prone to flashback. Instead, the results indicate that the axial momentum of the hydrogen jets is reduced at lean conditions, and this change increases the flashback propensity of the burner. As pointed by Freitz [7], combustion induced vortex breakdown is very sensitive to the momentum distribution in the vortex core, and this mechanism is known to be responsible of flashback in many swirl-stabilized premixed combustors [15]. The experimental results suggest the stability of the hydrogen flames is mainly governed by the level of axial momentum, and this has a dominant effect over the mixing quality and equivalence ratio fluctuations. This hypothesis motivates the present study where a perfectly premixed assumption is made for two stable operating points and the flames are investigated using large-eddy simulation (LES).

The use of LES for the prediction of complex phenomena in gas turbine combustors has been successful in many scenarios [40 10], although it poses several challenges from the physical and numerical point of view. The flow conditions are subjected to large disparity in length and time scales with large density variations caused by chemically reactive turbulent layers. While the application of LES has been widely used for describing self-excited instabilities and flame dynamics in gas turbine applications [39], the use of reacting LES at high Reynolds numbers including detailed chemistry near flahsback are more scarce. Sommerer et al. [28] studied combustion instabilities and flashback propensity of a lean premixed prevaporized combustor. They focused on the correlation between the central recirculation zone induced by the vortex breakdown and the upstream flame propagation. Tangermann and 
Pfitzner [31] conducted numerical investigations of the influence of the combustion models on the prediction of combustion-induced vortex breakdown. For this, they used reduced chemistry with thickened flame models at less stringent conditions.

The current work is focused on the characterization of the reacting flow field of a swirlstabilized combustor operated with hydrogen in lean premixed conditions in the context of large-eddy simulation (LES). This combustor is operated at relatively high Reynolds number ( $R e=75000$ based on the mixing tube flow conditions) at low Damkhöler number, and a combustion model based on flamelet modelling is employed. Particular attention is given to the characterization of the flow during stable operation until the equivalence ratio is near flashback. The modelling strategy is based on the assumption of a perfectly premixed mixture. This hypothesis is suggested from the experimental observations and is verified during stable operation of the burner by means of LES. Numerical data in combination with experimental PIV and POD are used for the analysis of the flames and model validation. The study includes an analysis of self-excited oscillations under inert and reacting conditions, and an assessment of the influence of axial momentum and combustion on the suppression of this hydrodynamic instability. A discussion of the flame structure and turbulent burning velocity is also presented.

The work structure is defined as follows. First, the LES modelling approach and numerical discretization is described. Secondly, a description of the experimental test rig and burner operation are provided along with a description of the flow conditions. An analysis of results are given subsequently that ends with some conclusions and directions for future work.

\section{Modelling approach}

2.1 Governing equations for large-eddy simulation

The governing equations describing the reacting flow field correspond to the low-Mach number approximation of the Navier-Stokes equations with the energy equation represented by the total enthalpy. A Favre-filtered description of the governing equations is followed to avoid modelling of terms including density fluctuations [23]. The governing equations are given by:

$$
\begin{gathered}
\frac{\partial \bar{\rho}}{\partial t}+\nabla \cdot(\bar{\rho} \widetilde{\mathbf{u}})=0 \\
\frac{\partial(\bar{\rho} \widetilde{\mathbf{u}})}{\partial t}+\nabla \cdot(\widetilde{\rho \mathbf{u} \widetilde{\mathbf{u}}})=-\nabla \bar{p}+\nabla \cdot\left[\bar{\rho}\left(\bar{v}+v_{t}\right)\left(2 S-\frac{2}{3}(\nabla \widetilde{u}) I\right)\right] \\
\frac{\partial(\bar{\rho} \widetilde{h})}{\partial t}+\nabla \cdot(\bar{\rho} \widetilde{\mathbf{u}} \widetilde{h})=\nabla \cdot\left[\bar{\rho}\left(\bar{D}+\frac{v_{t}}{S c_{t}}\right) \nabla \widetilde{h}\right]
\end{gathered}
$$

where $\bar{\rho}, t, \widetilde{\mathrm{u}}, \bar{p}, \bar{v}, \widetilde{h}$ and $\bar{D}$ represent the density, time, velocity vector, pressure, kinematic viscosity, total enthalpy and thermal diffusion coefficient respectively. Heating due to viscous forces is neglected in the enthalpy equation and the unresolved heat flux is modelled using a gradient diffusion approach [19]. The formulation is closed by an appropriate expression for the subgrid-scale or eddy-viscosity $v_{t}$ that in this study is defined by the closured proposed by Vreman [37] with a model constant of $c_{s}=0.1$. The viscous stress 
tensor is defined based on Stoke's assumption and the turbulence contribution is determined by the use of the Boussinesq approximation [23], in which $S=\frac{1}{2}\left[\nabla \widetilde{u}+(\nabla \widetilde{u})^{T}\right]$ and $I$ are the strain and the identity tensor respectively. A unity Lewis number assumption has been made to simplify the multicomponent transport in the governing equations. This assumption is made based in two facts: (1) the Reynolds number of the flame is relatively high and turbulent transport is expected to dominate over molecular transport, and (2) the experimental findings suggest that the fundamental physical mechanism behind the stabilization of the flames is the axial momentum achieved in the premixing section that provide resistance to flashback and is expected to be little influenced by preferential diffusion processes [25]. Turbulent Schmidt and Prandtl numbers are both set constant with value of 0.7.

\subsection{Combustion model}

The flow conditions considered in this study include the oxidation of a hydrogen/air mixture a preheated conditions in a turbulent flow field at two equivalence ratios. The laminar flame speed $s_{L}$ and flame thickness $\delta_{L}$ at these pre-heated conditions of the burner operation are shown in Fig. 1 a) as function of the equivalence ratio. It shows the flame thickness is relatively constant from an equivalence ratio $\phi=0.4$ suggesting that the same mesh resolution could be used in flame resolving simulations. This will be further discussed in the next subsection. Note that the flame thickness is of the order of $0.3 \mathrm{~mm}$, which is substantially smaller than those of hydrocarbon fuels. Nevertheless, it is observed that the flame speed changes more abruptly with local variations of the equivalence ratio, although this effect will be neglected in the present study by the perfectly premixed assumption. It also shows the wide flammability range that characterizes hydrogen flames and indicates the proximity of the lean limit at around $\phi=0.2$. This limit underlines the risk of extinction and blow-out during the operation of the burner when it approaches to flashback conditions.

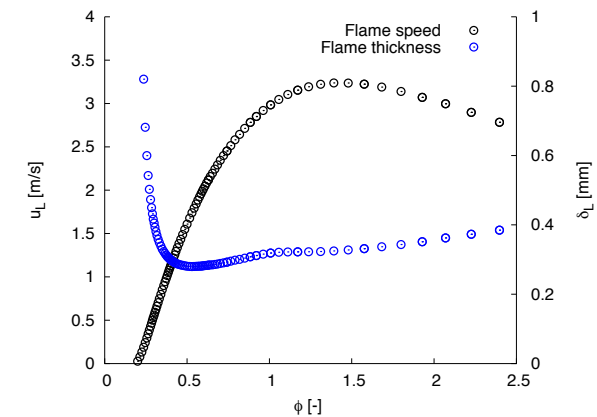

(a)

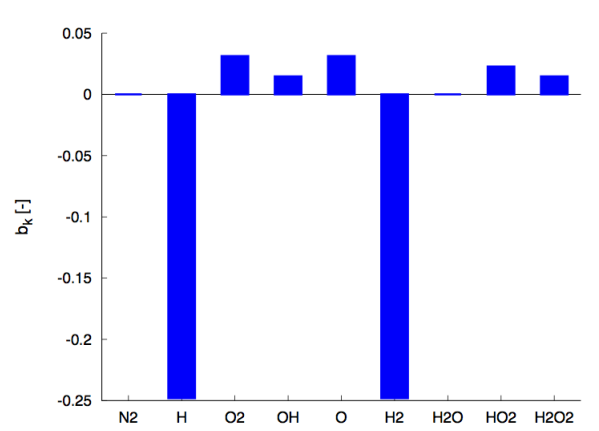

(b)

Fig. 1: (a) Laminar flame parameters of a hydrogen/air flame at preheated conditions $\mathrm{T}=$ $453 \mathrm{~K}$ and atmospheric pressure and (b) coefficient constants $b_{k}$ for $Y_{c}$ definition with CSP method.

The combustion process is assumed to take place in the flamelet regime for all conditions tested in this study. The description of the reacting flow in this regime is based on the 
assumption that chemical time scales are much faster than turbulent time scales, and hence, the thermochemical properties of the flame can be described from a precomputed flamelet database. The flamelet database is based on the tabulation of laminar premixed flamelets at constant equivalence ratio and uses the chemistry from the full San Diego mechanism [17], which demonstrated excellent performance for predicting hydrogen flames in a variety of flow conditions [18,19]. For the present combustion model, a controlling variable based on a reactive scalar can be used to couple the chemical states with the fluid flow. This controlling variable can be understood as a progress variable $Y_{c}$ that is used to describe the thermochemical state from an unreacted mixture to a fully reacted mixture. This indicates that any property from the manifold i.e. species mass fractions, reaction rates or transport properties, are unique function of $Y_{c}$ such that $\psi=\psi\left(Y_{c}\right)$ where $\psi$ is any property from the manifold. In this study, different definitions of the progress variable were examined to obtain a suitable definition valid for the two equivalence ratios. Classical definitions of the progress variable for hydrogen flames were compared with the one provided by the Computational Singular Perturbation (CSP) method [9] In the CSP method, the progress variable is defined as:

$$
Y_{c}=\sum_{k}^{N}\left(b_{k} Y_{k}\right)
$$

where $b_{k}$ is provided by the CSP reduction technique and $Y_{k}$ are the mass fractions of the selected species. The weights $b_{k}$ obtained by the CSP method at these two equivalence ratios is shown in Fig. 1] b). For numerical reasons [6], it is more convenient to define a scaled progress variable $c$, which can be defined as:

$$
c=\frac{Y_{c}-Y_{c}^{0}}{Y_{c}^{e q}-Y_{c}^{0}}
$$

where $Y_{c}^{0}$ and $Y_{c}^{e q}$ are the values of the progress variable of the unreacted mixture and at equilibrium conditions respectively. The distribution of the source term as function of the scaled progress variable $c$ using different definitions of $Y_{c}$ is shown in Fig. 1 at two equivalence ratios. The plots indicate the use of CSP leads to a more uniform distribution of the source term in $c$ space compared to other definitions, though it is not so different from using the mass fraction of $\mathrm{H}_{2} \mathrm{O}$ at these operating conditions.

Considering the application of this flamelet combustion model to premixed combustion in LES, a filtered scaled progress variable $\widetilde{c}$ needs to be defined. The filtering operation is done by:

$$
\widetilde{c}=\int_{0}^{1} \psi(c) \widetilde{P}\left(c^{*}\right) d c^{*}
$$

where $\widetilde{P}$ is a Fave-averaged probability density function (PDF) that is specified with a presumed-shape PDF based-on a $\beta$-function [6]. This PDF is constructed from the filtered progress variable $\widetilde{c}$ and the subgrid variance $\widetilde{c^{\prime \prime 2}}=\widetilde{c c}-\widetilde{c c}$. A closure for the subgrid scale variance is provided by the solution of the transport of $\widetilde{c^{\prime \prime 2}}$ following Domingo and Vervisch [6]. 


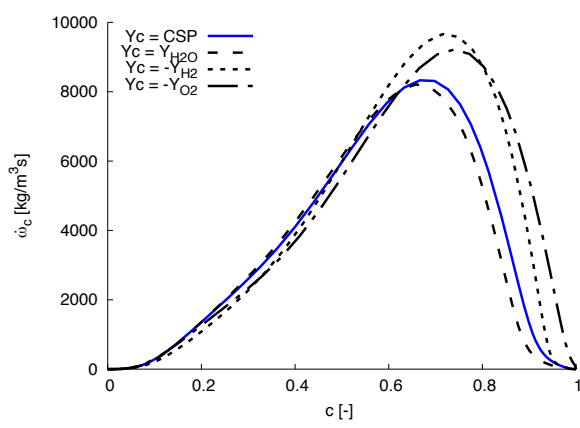

(a)

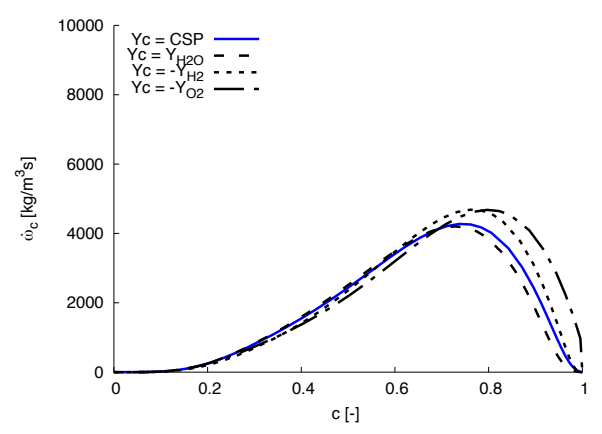

(b)

Fig. 2: Progress variable $Y_{c}$ definition for hydrogen/air combustion at preheated conditions $(\mathrm{T}=450 \mathrm{~K})$ and atmospheric pressure $(\mathrm{p}=1 \mathrm{bar})$ : (a) distribution of the source term at $\phi=$ 0.6 , and (b) distribution of the source term at $\phi=0.4$.

The chemical state of the perfectly premixed flame in the LES framework is ultimately described by the two controlling variables: $\widetilde{c}$ and $\widetilde{c^{\prime \prime 2}}$, so the governing equations describing the chemical evolution of the flame are given by:

$$
\begin{aligned}
\frac{\partial(\bar{\rho} \widetilde{c})}{\partial t}+\nabla \cdot(\bar{\rho} \widetilde{\mathbf{u}})=\nabla \cdot[ & \left.\bar{\rho}\left(\bar{D}+\frac{v_{t}}{S c_{t}}\right) \nabla \widetilde{c}\right]+\overline{\dot{\omega}}_{c} \\
\frac{\partial\left(\bar{\rho} \widetilde{c^{\prime \prime 2}}\right)}{\partial t}+\nabla \cdot\left(\bar{\rho} \widetilde{\mathbf{u}} \widetilde{c^{\prime \prime 2}}\right)= & \nabla \cdot\left[\bar{\rho}\left(\widetilde{D}+\frac{v_{t}}{S c_{t}}\right) \nabla \widetilde{c^{\prime \prime 2}}\right] \\
& +2 \bar{\rho}\left(\bar{D}+\frac{v_{t}}{S c_{t}}\right)\left|\nabla \widetilde{c^{\prime \prime 2}}\right|^{2} \\
& +2\left(\overline{c \dot{\omega}}-\widetilde{c} \dot{\dot{\omega}_{c}}\right) \\
& -\bar{\chi}_{c}
\end{aligned}
$$

where $\bar{\chi}_{c}$ represents the scalar dissipation rate and $\bar{\omega}_{c}$ is the source term of the progress variable. The scalar dissipation rate is composed by the resolved and unresolved parts, which are given by:

$$
\bar{\chi}_{c}=2 \bar{D}|\nabla \widetilde{c}|^{2}+\chi_{c}^{s g s}=2 \bar{D}|\nabla \widetilde{c}|^{2}+\frac{C_{d}}{\tau_{t}} \widetilde{c^{\prime \prime 2}}
$$

where $\tau_{t}$ is a turbulent time scale obtained from $1 / \tau_{t}=\varepsilon_{s g s} / k_{s g s}$ as proposed by Ventosa et al. [36]. The subgrid scale component assumes $\widetilde{c}$ is a reactive scalar and its gradient is influenced by chemistry, so that $\chi_{c}^{s g s}$ is closed with the combined linear relaxation hypothesis and bimodal limit closure [6].

\subsection{Numerical discretization}

The governing equations (1), 2), (3), (7), and (8) are solved by means of a low-dissipation finite-element method implemented into the code Alya [35].The convective term is dis- 
cretized using an extension to variable density flows of the scheme recently proposed by Charnyi et al. [4], which conserves linear/angular momentum and kinetic energy at the discrete level. Second-order spatial discretizations are used. In order to use equal-order elements, numerical dissipation is introduced only for the pressure stabilization via a fractional step scheme [5]. The set of equations is integrated in time using a third-order RungeKutta explicit method combined with an eigenvalue-based time-step estimator [34]. This approach has been shown to be significantly less dissipative than traditional stabilized FEM approach [16]. Scalar equations are solved by a third-order Runge-Kutta explicit method combined with an ASGS method [2].

\section{Setup}

This section describes the experimental test rig and computational setup proposed to investigate the stable flames when operated with hydrogen at medium axial air injection [25]. It also includes a description of the test cases and an assessment of resolution for the numerical simulations.

\subsection{Experimental test rig}

The test case for analysis is depicted in Fig. 3. a) and corresponds to a technically premixed burner consisting of two oxidizer inlets, a radial swirler and an orifice on the central axis fed by a shared plenum. The ratio of the axial jet over the non-swirling flow is determined by the pressure losses of both components and thus the intensity of the axial jet is adjusted by the diameter of the orifice, being $s=8.0 \mathrm{~mm}$ in our case. The axial jet, added through a hollow cone, and the swirled flow, added through the swirl generator, enter a mixing tube of $60 \mathrm{~mm}$ length and diameter of $\mathrm{D}=34 \mathrm{~mm}$. At the end of the mixing tube, the mixture flows over an expansion cross-section with a diameter ratio of 3.1 into the combustion chamber.



(a)

(b)

Fig. 3: (a) Experimental configuration and (b) Combustion chamber sketch with the measurement stations for validation with PIV. 
The combustion chamber length was reduced in the simulations to 8.0D in order to minimize the number of computational cells in the far upstream flow region that has less interest. The fuel is injected into the mixing tube through 16 injection holes of $1.6 \mathrm{~mm}$ diameter located on the annular ring between the cone and the swirler inlets. Further information is given in references [24 32]. An sketch of the combustion chamber is also presented in Fig. 3. b) to have a visual representation of the flame location and recirculation zones along with the location of the measurement planes for quantitative comparison with the PIV.

\subsection{Test cases}

The computational cases considered in this study are presented in Fig. 4 by red points on top of the stability map of the burner for a medium axial air injection. The three cases have a Reynolds number $\mathrm{Re}=75000$ with pre-heated air at $\mathrm{T}_{\text {air }}=453 \mathrm{~K}$ and hydrogen coming at $\mathrm{T}_{\mathrm{H} 2}=320 \mathrm{~K}$. The first case CTR1 corresponds to inert conditions without fuel injection and it is used not only for validation purposes, but also for the evaluation of the impact of heat release on the dynamics of the flow in this burner concept. The other two cases (CTR2 and CTR3) correspond to reacting flow calculations for which experimental data is available. Before igniting the flame in the simulation, a statistically steady state cold flow of both cases is reached. Hence, it is possible to analyze the influence of the heat release onto the flow field. As shown in Fig 4 , it is known from the experimental studies that these two cases have a stable flame. Further reduction of the equivalence ratio would lead to transitional cases at the onset of flashback and close to the lean blowout limit (LBO) [24], which are left for future work. The definition of the latter two cases and the comparison with the technically premixed assumption is discussed in the next subsection.

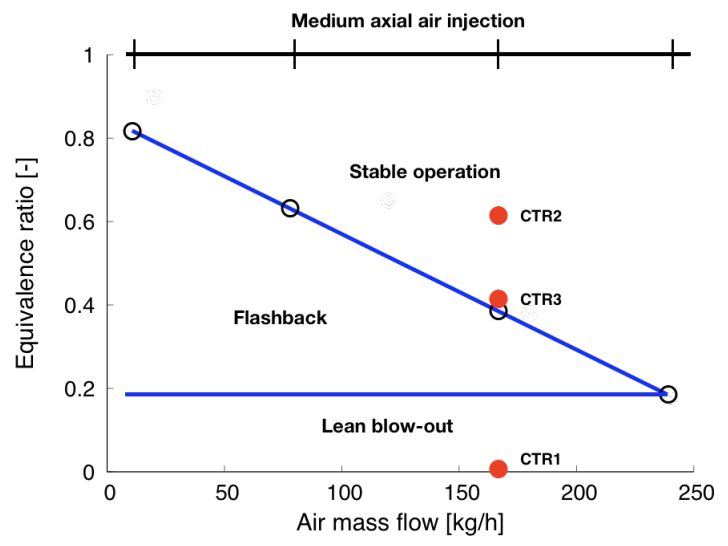

Fig. 4: Stability maps and selected computational cases for burner operation.

A summary of the computational cases included in this study is given in Table 1 , where $\phi, Z_{g}$, and $J$ represent the equivalence ratio, global mixture fraction, and axial momentum of the fuel jets respectively. The momentum ratio $J$ is defined in accordance to Reichel and Paschereit [24] as:

$$
J=\frac{\rho_{\text {fuel }} u_{\text {in } j, \text { fuel }}^{2}}{\rho_{\text {air }} u_{0, \text { air }}^{2}}
$$


where $u$ is the axial bulk velocity at the fuel injectors $u_{i n j, f u e l}$ and the equivalent axial velocity at the mixing tube from the air system supply $u_{0, \text { air }}$ respectively.

Table 1: Computational cases

\begin{tabular}{cccc}
\hline Case & $\phi$ & $\mathrm{J}$ & $Z_{g}$ \\
\hline CTR1 & 0.0 & 0.0 & 0.0 \\
CTR2 & 0.6 & 2.5 & 0.0171 \\
CTR3 & 0.4 & 1.1 & 0.0115 \\
\hline
\end{tabular}

\subsection{Assessment of resolution}

In order to evaluate the effects of mesh resolution on the LES results that will be presented in the next section, a mesh convergence analysis with different grids for CTR1 was made. The Kolmogorov length and time scales were computed at different locations of the burner. The results from the different grids are omitted here for simplicity, but an estimation of the Kolmogorov scale on the final mesh scaled with the mesh length scale $\Delta$ are shown in Fig 5 across the length of the mixing tube and combustion chamber. Note the data is normalized with the mixing tube length $L$, so the combustion chamber is located at $L=1$. Details of the mesh resolution used for all the computational cases is summarized in Table 2 .

Table 2: Mesh resolution

\begin{tabular}{ccc}
\hline Component & $\Delta[\mathrm{mm}]$ & $y+$ \\
\hline Fuel injection & 0.20 & $y^{+}<100$ \\
Mixing tube & 0.36 & $y^{+}<10$ \\
Combustion chamber & 0.45 & $y^{+}<50$ \\
\hline
\end{tabular}

The results indicate that the flow shows a large disparity of scales in the mixing tube and combustion chamber. The plot Fig 5 a) shows a reduction of length scales as the mixing tube starts, see $\mathrm{x} / \mathrm{L}=0.0$, which corresponds to the location where the mixing between the axial air injection and swirler takes place. In this region, high circumferential velocities are formed due to swirling motion that interact with the axial air injection resulting in high levels of turbulence and mixing. In this region, the fuel mixes with the axial jet promoting high levels of turbulence and reducing the size of the small scales. The length scales in the combustion chamber can be distinguished in red from $\mathrm{x} / \mathrm{L}>1.0$. The smallest scales are found at the exit of the mixing tube before the vortex breakdown is developed at around $\mathrm{x} / \mathrm{L}$ $=1.5$. Further downstream, a recirculation zone is formed with negative axial velocity and the turbulence is dissipated by the vortex breakdown and the flow expansion increasing the size of the scales as can be seen from $x / L>2.3$. The results indicate the proposed mesh is appropriate for this problem as the resolution available in the mixing tube and combustion chamber is in the order of 5 to 10 times the Kolmogorov length scale $l_{k}$. In terms of time scales, the time step size is set to $\mathrm{CFL}=0.95$ and it is of the order of $10^{-7}[\mathrm{~s}]$, which results 
in smaller time-steps than the Kolmogorov time scale $t_{k}$ as the high velocity injection is resolved through the injection holes. While this analysis is done with the data of the inert
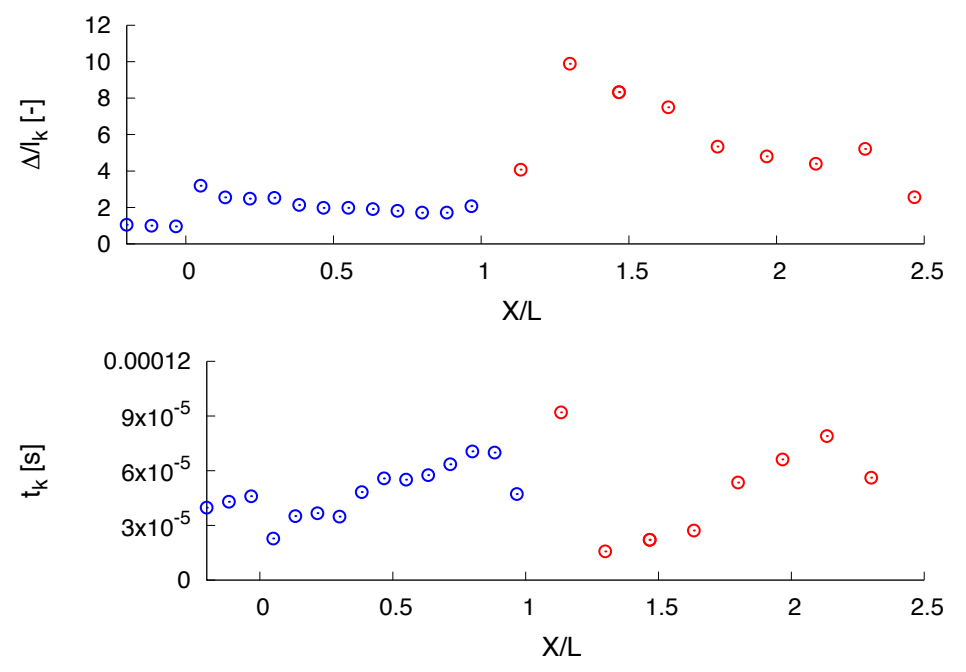

Fig. 5: Kolmogorov length (top) and time (bottom) scales along the centreline for the mixing tube (blue) and combustion chamber (red).

case CTR1, it can be used as a priori estimation of the level of resolution available for the reacting cases. The structures in the mixing tube will follow a similar evolution to the inert case. Moreover, the resolution requirements in the combustion chamber are favoured by the reduction of the overall turbulence level due to the increase in viscous diffusion caused by thermal heating. Considering the resolution available to compute the reacting layer has a ratio of $\Delta / \delta_{L} \approx 1.5$, it suggests the modelling approach proposed here based on presumedshape PDF will be valid for this level of resolution [6].

\section{Results}

This section describes the numerical results obtained with the modelling strategy proposed in the previous section. First, a description of the flow dynamics for the inert case with comparison of the PIV data from experiments is presented. Secondly, an assessment of the perfectly premixed condition using mixing data from technically premixed simulations [20] is given with focus on the flow across the mixing tube. Note that measurements can only be taken in the combustion chamber, and numerical data in this part of the burner is of primary interest to understand the flow conditions before ignition and ultimately prevent the formation and development of flashback in this burner. Finally, the study of the reacting flow field of the two stable operating points (CTR2 and CTR3) using a perfectly premixed assumption is presented. 


\subsection{Inert flow conditions}

Mean flow field

The characteristics of the flow field of the inert case including the LES and the experimental data is discussed in this section. The distribution of mean axial and radial velocities in the combustion chamber for the simulations and experiments are shown in Fig. 6 using contour plots. A good level of correlation between the LES and PIV data is found. It is noted that the LES plot shows mean values that still require further time-averaging as the flow is characterized by a large disparity in time and spatial scales. To obtain a fully converged first-order statistics in the three-dimensional domain would require a larger interval for integration. The present simulation is time-averaged for 2.5 flow-through $\tau=\langle U\rangle / V$, where $\langle U\rangle$ is the mean velocity integrated along the volume of the mixing tube and combustion chamber and $V$ is the volume of the two components. The axial velocity is well predicted with the correct level of penetration, central and corner recirculation zones. The radial velocity is also in good agreement with the correct spreading and penetration.

The data has been further compared at five streamwise locations (see Fig. 3 (b) for exact locations) for mean and fluctuations of the axial and radial velocities and this is shown in Fig. 7. In order to have more statistically converged data, the LES results have been azimuthally-averaged. Overall, the comparison is satisfactory at all locations and highlights the good predicting capabilities of the physical and numerical approach.

\section{Flow dynamics}

The inert flow field (CTR1) in the combustion chamber reveals a strong characteristic frequency at approximately $1100 \mathrm{~Hz}$, which can be found in the PIV as well as in the LES data. The frequency is slightly over-predicted in the LES but generally in the same order of magnitude. The observation point for the spectra in Figure 8 is located in the shear layer of the recirculation zone shortly downstream of the mixing tube exit. The characteristic frequency corresponds to the Precessing Vortex Core (PVC) [29]. The PVC is a well-known global flow instability, which can arise in swirling flows underlying vortex breakdown. The Kelvin-Helmholtz instabilities in the inner shear layer induce vortexes that are convected downstream. Due to the inverse axial velocities in the recirculation zone, these vortexes trigger a feed back loop and a precessing motion of the recirculation zone's stagnation point. Even though the flow field is highly turbulent, these instabilities form structures with a high degree of coherence. Thus, they can be well visualized by Proper Orthogonal Decomposition (POD) [1], which is a method of empirical mode decomposition. The POD modes decompose the flow field in a way to optimally resolve the maximum turbulent kinetic energy in a arbitrary subset of first modes. Hence, the first two modes are corresponding to the coherent structures resolving the largest amount of fluctuation energy, in this case the PVC modes. Figure 9 is showing the two modes which reflect the structure of the PVC, calculated from the instantaneous fluctuations of the radial velocity in the combustion chamber. In both cases, the experimental (top row) as well as the simulated (bottom row), one can easily identify the dominating PVC structure. Thereby, the first and second mode reveal a periodic behavior that occur with the characteristic frequency. They are phase shifted about a quarter period in time and space. Thus, their superposition leads to a downstream propagation of the structures, i.e. the vortexes, which are induced in the shear layer. Even though the modes are axis-symmetric in the plot, the flow field is not. The vortex arises alternately in the left and right shear layer. In three dimensions the PVC reflects a single-helical co-rotating 


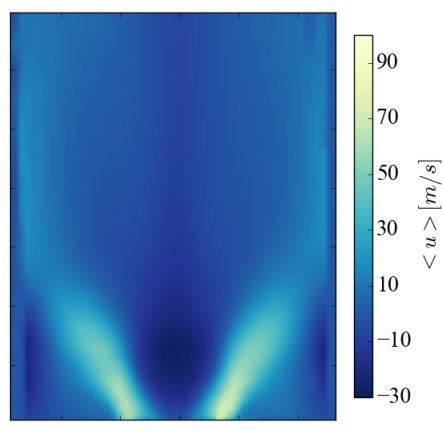

(a)



(c)

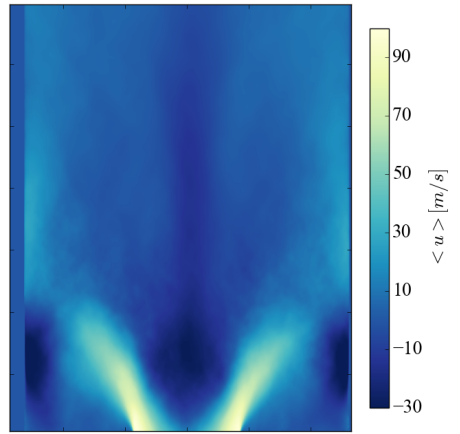

(b)

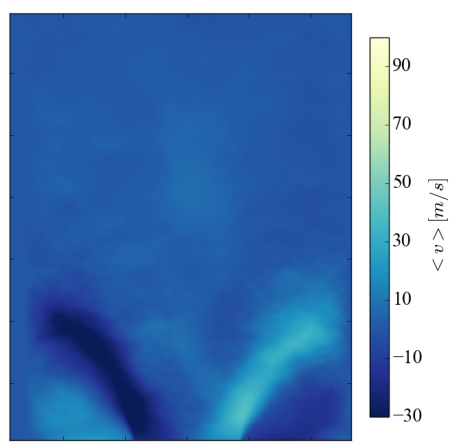

(d)

Fig. 6: Comparison of the numerical simulations with the experimental PIV data for CTR1: (a) PIV mean axial velocity and fluctuations, (b) LES mean axial velocity, (c) PIV mean radial velocity and (d) LES mean radial velocity. Solid line: LES present work, symbols: experimental data.

vortex structure, which is synchronized with the precession of the recirculation zone. The axis-symmetry in the two-dimensional mode shape appears just due to the different signs of the radial velocity fluctuation on both sides.

Comparing the top and bottom row, Fig. 9 shows that the LES is able to precisely reproduce the PVC structure. The modes of the LES data appears more blurry. However, this stems from the low number of snapshots (only 20) used for the calculation.

The above described case CTR 1 simulates only the air flow without any fuel injection $(J=0)$. The air flow in the mixing tube has axial as well as tangential momentum due to the swirl generator. In order to enable premixed combustion, hydrogen is injected at the upstream end of the mixing tube. This is done by 16 axial non-swirling jets. Thus, they impose an additional axial momentum to the flow, which lowers the swirl number. The swirl number is the ratio of tangential to axial momentum and is calculated in accordance with [33] by Equation 13. Figure 10 (left) shows that the additional axial momentum at $J=$ $2.5(\phi=0.6)$ decreases the swirl number by more than $10 \%$ compared to the case without 



(a)


(b)

Fig. 7: Comparison of the numerical simulations with the experimental PIV data for CTR1: (a) mean axial velocity and fluctuations, (b) mean radial velocity and fluctuations. Solid line: LES present work, symbols: experimental data.

fuel, i.e. CTR1. The swirl number has a significant effect on the PVC as shown in Fig. 10 (right). Here the spectra of cold flow simulations reveal that the energy of the characteristic peak is decreasing with increasing fuel momentum. While with a fuel momentum of $J=1.1$, i.e. $\phi=0.4$, the peak is still detectable and the POD modes also proof the PVC in the flow field, there is no characteristic frequency at $J=2.5$, respectively $\phi=0.6$. Hence, the $\mathrm{PVC}$ is suppressed by the additional fuel momentum at high $J$. A similar effect was already 


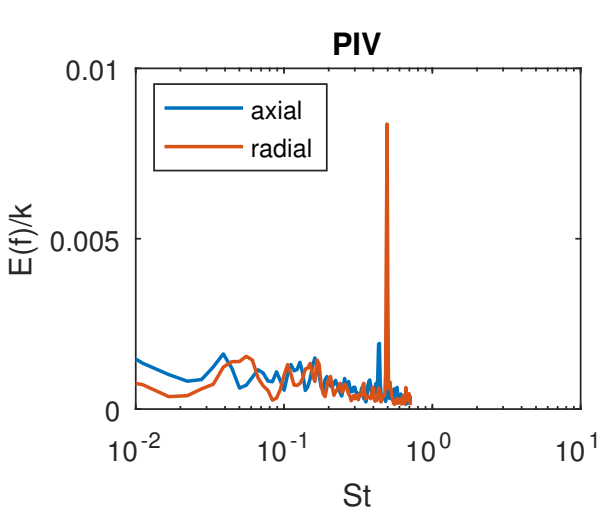

(a)

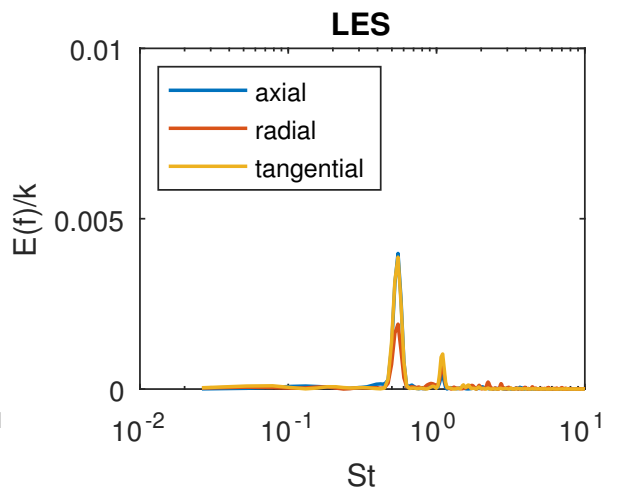

(b)

Fig. 8: Power spectral density of the velocity fluctuation at an observation point in the combustion chamber ( $x=10 \mathrm{~mm}, r=15 \mathrm{~mm}$ ) at PIV (a) and CTR1 (b).

described by Terhaar et al [33], who varied the swirl number by increasing the axial air injection of the burner and thereby suppressed the PVC.

$$
S_{n}=\frac{G_{t}}{R \cdot G_{a x}}=\frac{2 \pi \rho \int_{0}^{R} u w r^{2} d r}{R \cdot 2 \pi \rho \int_{0}^{R}\left(u^{2}-0.5 w^{2}\right) r d r}
$$

\subsection{Assessment of the perfectly premixed condition}

From the experimental studies of this burner [24,25], it was observed that the flow is highly influenced by the axial momentum introduced by the fuel injectors. In fact, this is found to be the principal mechanism for flame stabilization in the combustion chamber that prevents the formation of flashback. The flow field in the inert case (CTR1) exhibits a deficit in axial velocity toward the center line across the mixing tube, which is corrected in the cases with fuel injection CTR2 and CTR3. The axial injection yields a more homogeneous radial distribution of axial velocity along the mixing tube and pushes the stagnation point further downstream. In order to evaluate the role of axial momentum over fuel homogeneity and equivalence ratio fluctuations, perfectly premixed flow conditions were set to study the stable flames. This parameter is of special interest in hydrogen combustion as the fuel has high volume flow due to its low density, and high velocities are expected to appear through the injection holes.

The perfectly premixed assumption is achieved by imposing the same total mass flow rate and equivalence ratio as that of the technically premixed experiment, so the thermal power is kept constant. The second requirement to be able to compare with the experiment is to have the same axial momentum in the fuel injection. The axial flow momentum is known to be a key parameter to control the flame dynamics and the flashback prevention in this burner [24,25]. Finally, the boundary conditions for the LES calculation in premixed conditions (see eq. (14) ) are set with the total flow rate from the technically premixed case, 



(a)
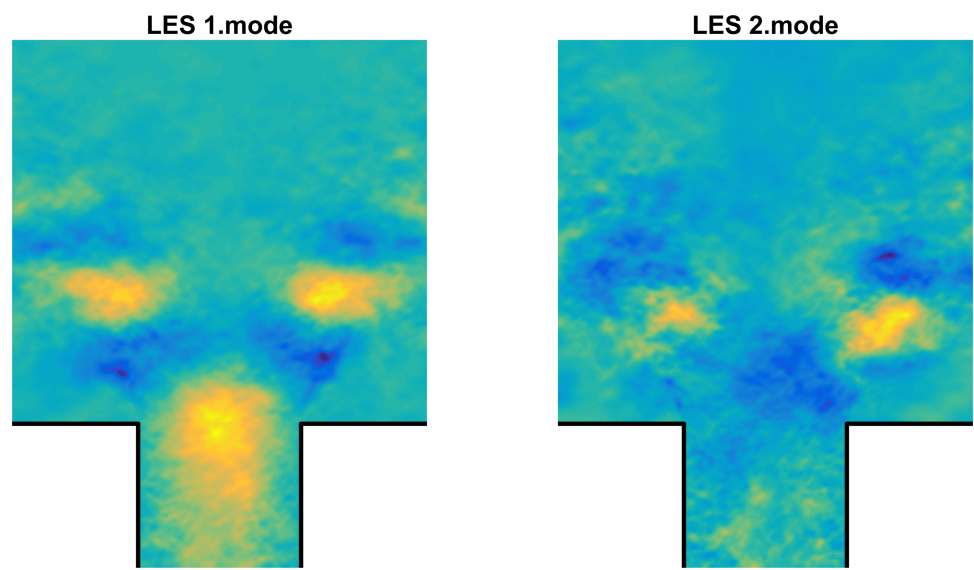

(b)

Fig. 9: Proper orthogonal decomposition of the radial velocity fluctuation in the combustion chamber for PIV (top) and CTR1 (bottom).

and injection holes flow rates coming from matching the axial momentum in technically and perfectly premixed conditions. This condition can be stated as:

$$
\begin{gathered}
J_{t p}=J_{p p} \quad \dot{m}_{t p}=\dot{m}_{p p} \\
\frac{\rho_{f u e l} u_{i n j, t p}^{2}}{\rho_{\text {air }} u_{0, t p}^{2}}=\frac{u_{i n j, p p}^{2}}{u_{0, p p}^{2}}
\end{gathered}
$$

where $u_{i n j, t p}, u_{0, t p}, u_{i n j, p p}$ and $u_{0, p p}$ refer to the axial injection velocity of the fuel and air in technically premixed " $t p$ " and perfectly premixed " $p p$ " conditions respectively. Providing the total flow rate in the burner, the mass flows for each component (fuel injection and air injection systems) can easily be obtained. Please note this calculation assumes the 16 injection holes distribute the flow rate homogeneously to the mixing tube, and this is not 

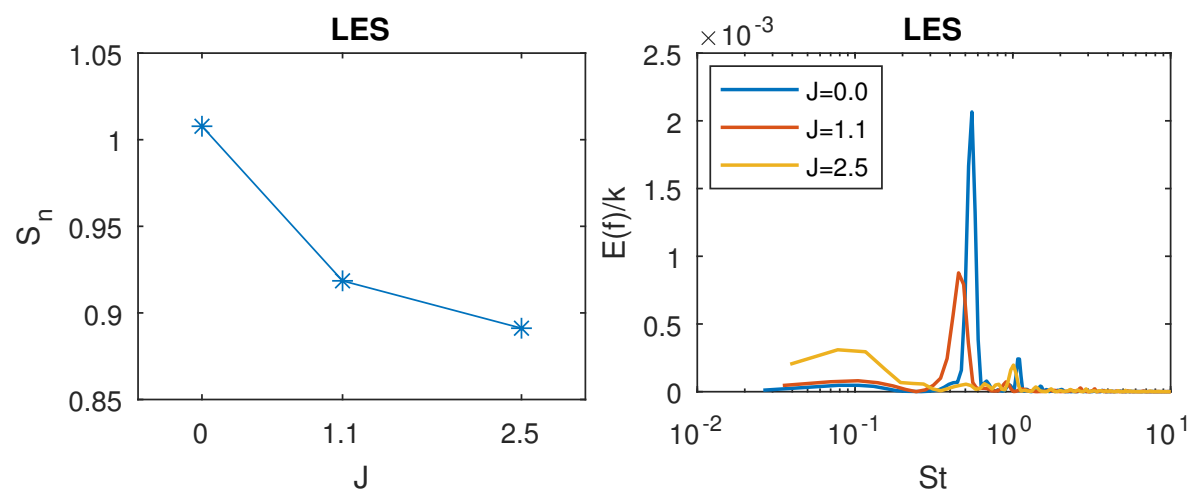

Fig. 10: Swirl number at the outlet of the mixing tube and power spectral density of the absolute velocity fluctuation at an observation point in the combustion chamber $(x=10 \mathrm{~mm}, r=$ $15 \mathrm{~mm}$ ) for the cold flow of all simulation cases.

exactly the case in the present burner. Evaluation of the injection holes using mean velocity fields suggest different flow rates between the holes with those closer to the injection pipes delivering larger flow rates. Nevertheless, for the description of the boundary conditions for LES this effect is neglected and the total mass flow rate coming from the two fuel ports is computed with this hypothesis. This is expected to have certain impact on the actual axial momentum of the burner, but as the flow is further developed along the mixing tube, the impact on the velocity distribution of the hydrogen-air mixture at the entrance of the combustion chamber should be small.

In order to evaluate the correct implementation of the boundary conditions for LES based on matching the axial momentum (see eq. (14)), an analysis of momentum fluxes along the mixing tube is presented in Fig. 11 for CTR2 and CTR3. It shows the evolution of the flux of axial and tangential momentum for the technically and perfectly premixed cases respectively. A good agreement is observed for the fluxes near the injection point $(x / L=0.15)$ for the two cases, though the correlation of the axial momentum close the mixing tube exit slightly deviates. Nevertheless, the differences are small. As the flame can be strongly impacted by the homogeneity of the fuel/air mixture, a comparison of the mixture fraction distribution between the technically and partially premixed simulation at three locations along the mixing tube is shown in Fig 12 A non-homogeneous distribution of hydrogen is observed for the technically premixed case at upstream locations. The fuel distribution peaks at the radial position where the injection holes are located. As the mixture evolves along the mixing tube, the mixing quality improves and a relatively uniform mixture fraction distribution exists when the flow enters in the combustion chamber. The evaluation of the perfectly premixed condition is finally assessed in the reacting flow analysis.

\subsection{Reacting flow conditions}

\section{Mean flow field}

The distribution of the velocity flow field for the reacting cases CTR2 and CTR3 is presented in this section for the LES and PIV in Figs. 13 and 14 respectively. The results indicate a 

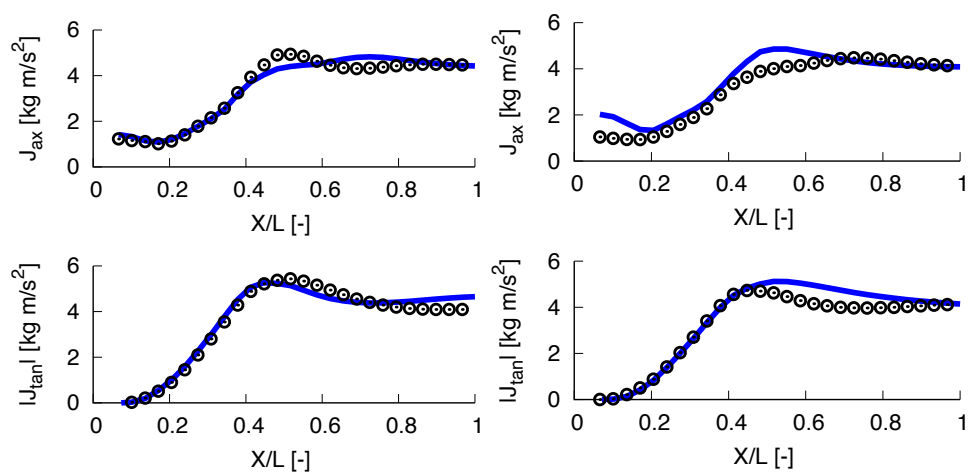

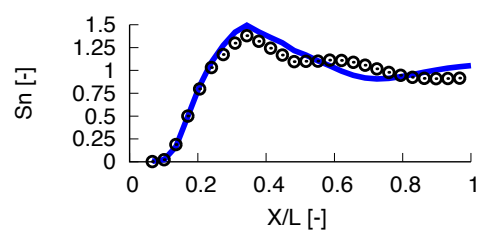

(a)

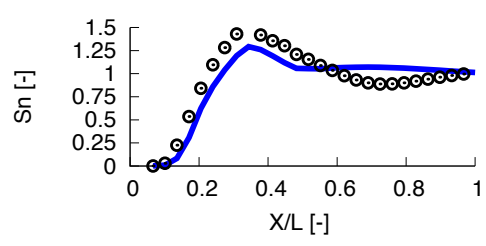

(b)

Fig. 11: Distribution of momentum fluxes: (top) flux of axial momentum, (middle) flux of tangential momentum and (bottom) swirl number. The plots correspond to (a) CTR2 and (b) CTR3. Solid line: perfectly premixed condition, symbols: technically premixed condition.
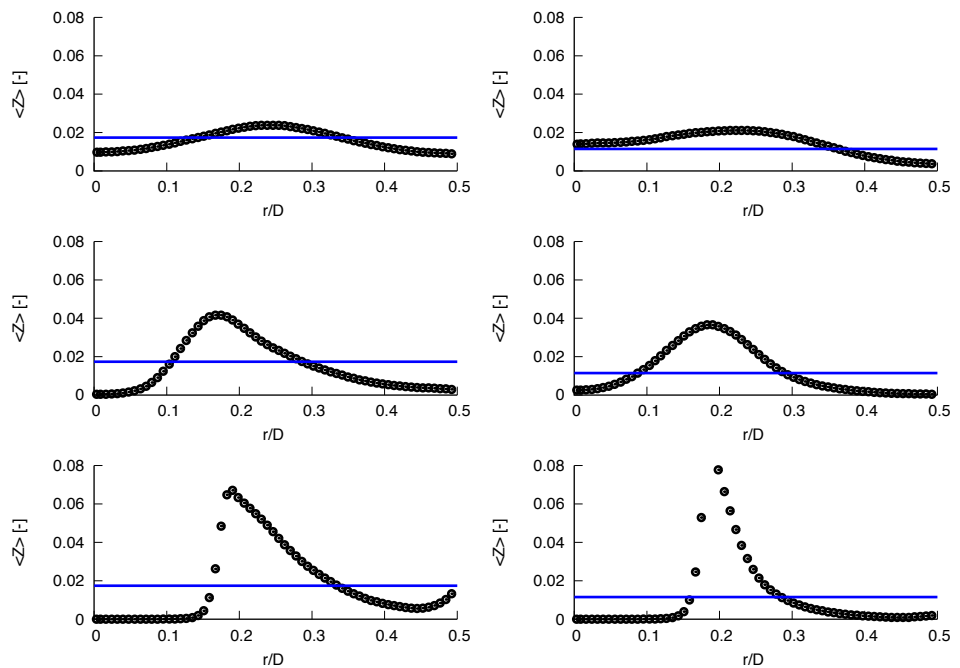

Fig. 12: Profiles of mixture fraction distribution at three axial locations for the technically and perfectly premixed cases. Bottom: $\mathrm{x} / \mathrm{L}=0.2$, middle: $\mathrm{x} / \mathrm{L}=0.4[\mathrm{~mm}]$ and top: $\mathrm{x} / \mathrm{L}=$ 0.9 for CTR2 (left) and CTR3 (right). Solid line: perfectly premixed condition, symbols: technically premixed condition. 
good agreement with the experimental data and validates the hypothesis of the perfectly premixed assumption considered by matching the axial momentum between the two cases with the same flow rate (see eq.(14) during the stable operation of the burner. The LES results are able to predict the transition of the vortex breakdown from bubble-type in the inert case to the cone-type in the reacting case, which is a fundamental aspect for flashback safety in this burner [25]. Comparing Fig. 13 to the inert case in Fig. 6 the central recirculation is shifted downstream with a wider spread of the annular jet due to the dilation caused by the heat release and the additional axial momentum introduced by the injection holes. The propagation of the vortex breakdown into the mixing tube is fundamentally induced by chemical reaction, and the burner is prone to combustion-induced vortex breakdown flashback [21].

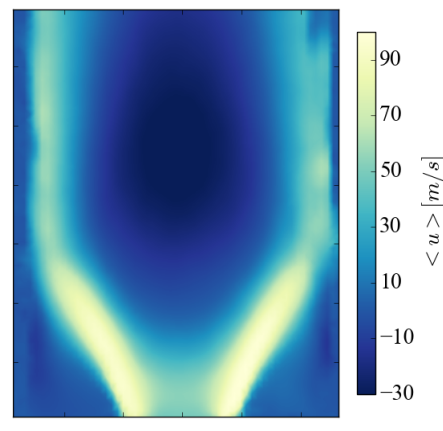

(a)

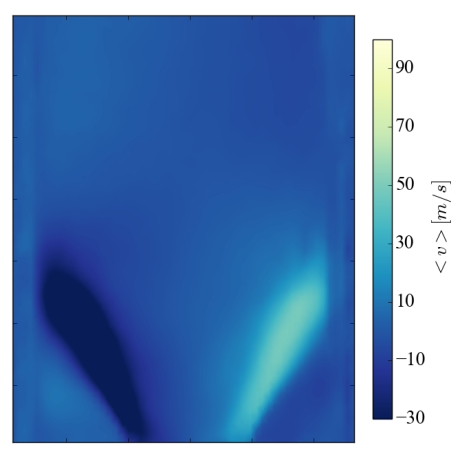

(c)

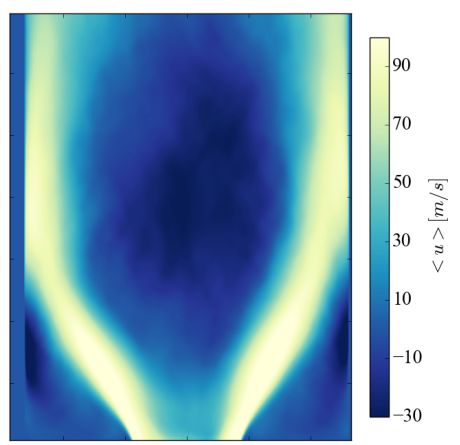

(b)

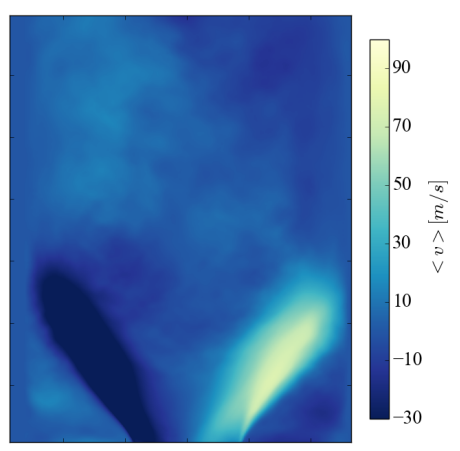

(d)

Fig. 13: Comparison of the numerical simulations with the experimental PIV data for CTR2: (a) PIV mean axial velocity, (b) LES mean axial velocity, (c) PIV mean radial velocity and (d) LES mean radial velocity.

A more quantitative comparison between the flow fields in the LES and experiments is presented in Figs. 15 and 16 for mean and fluctuating velocities. The plot shows the LES 
matches well the velocity deficit at the upstream location where the flame is stabilized and the correlation with the PIV also extends to the formation of the central recirculation and the spreading angle. The fluctuations are also in good agreement with the experimental data except for the radial velocity at the most upstream location, where the second-order statistics for this velocity component are subjected to lower frequencies and might require further integration time. This proves this technically premixed burner can be assumed to operate in perfectly premixed conditions during stable operation.



(a)

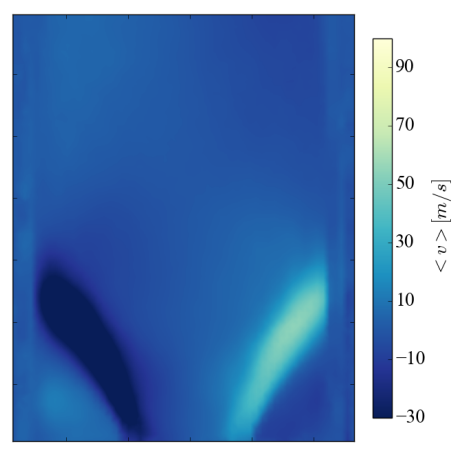

(c)

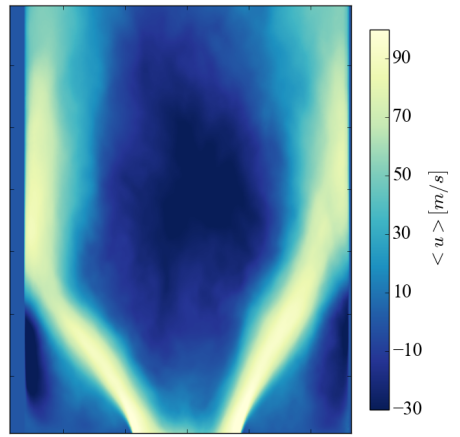

(b)

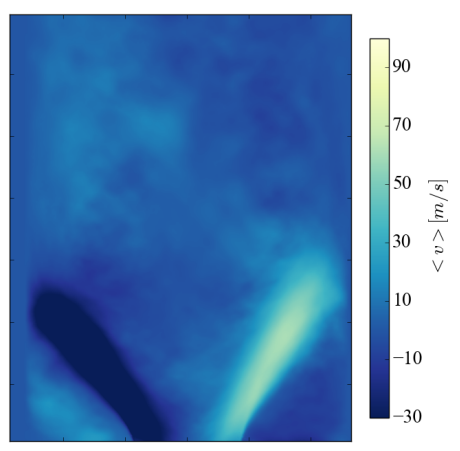

(d)

Fig. 14: Comparison of the numerical simulations with the experimental PIV data for CTR3: (a) PIV mean axial velocity and fluctuations, (b) LES mean axial velocity, (c) PIV mean radial velocity and (d) LES mean radial velocity. Solid line: LES present work, symbols: experimental data.

The agreement for both flames is satisfactory at all axial locations, though CTR2 seems to have better correlation at all measurement locations than CTR3. The LES results for CTR3 tend to overpredict the velocity deficit after the first measurement station and suggests the dynamics of the flame in this region exhibit certain dynamics that the simulations can not predict accurately. While the axial velocity is well predicted at the first measurement station, 
the radial velocity is overpredicted (see Fig. 16. This is highly influenced by the premixed section, but also the flow dilation caused by the heat release. The flame is stabilized few millimeters downstream of the burner, and the dynamics of the reacting layer have a strong influence on the velocity field in this region. At further downtream locations, the correlation with the PIV is fully recovered and the LES is capable of predicting the central recirculation and reverse flow accurately. It is concluded that as the flame approaches flashback conditions, the perfectly premixed assumption might not hold any longer and equivalence ratio variations can start playing a more important role.

\section{Flow dynamics}

The dynamics of the flow structures for the inert and reacting cases can be analyzed by the examination of the instantaneous flow through the use of the Q-criterion proposed by Hunt et al. ([12]). The Q-criterion is constructed from the second invariant of the velocity gradient tensor, which is decomposed into a symmetric (rate-of-strain, $\mathbf{S}$ ) and a skew-symmetric (rate-of-rotation, $\omega$ ) component. The second invariant of the velocity gradient tensor is defined as:

$$
Q=\frac{1}{2}\left(\|\omega\|^{2}-\|\mathbf{S}\|^{2}\right)
$$

The Q-criterion defines an eddy structure as a region with positive second invariant $(Q)$, which means regions where rotation dominates over strain. A visualization of instantaneous Q-iso-contours for the different cases is shown in Fig 17. A first inspection of the figure reveals the large quantity of small scales in the combustion chamber and the fundamental differences between the inert case CTR1 and reacting cases CTR2 and CTR3. The inert case shows a large disparity of length scales and results in a flow with larger spreading angle and less penetration. The same effect can also be distinguished between CTR2 and CTR3 where the thermal heating is lower for the low equivalence ratio case. The Q-contours shows the formation of helicoidal-like structures that are developed from the outer layer and wrinkle the flame front in the reacting cases.

As pointed out in the inert flow section, the cold flow of CTR3 reveals a flow instability, named PVC. This flow structure appears periodically and is determined by a characteristic frequency. Figure 18 (a) shows this frequency for the cold case as a strong peak in the power density spectrum of the absolute velocity fluctuation. On the other hand, the flow with heat release in the combustion chamber does not have a characteristic frequency. The same counts for the POD modes, which are similar to the ones in Figure 9 in the isothermal, but without any coherence in the reacting flow field. Thus, the heat release suppresses the occurrence of the PVC. The heat release of the flame alters the density field in the combustion chamber, which has an influence on the stability behavior in the shear layers. The PVC in the isothermal case emerges from a global flow instability in the recirculation zone, which might be damped in the reacting case. This damping effect was extensively investigated by Oberleithner et al. [22] using linear stability analysis. He found that not the altered velocity field, but strong density gradients close to the origin of the global instability are responsible for the suppression of the PVC. Accordingly, the existence of the PVC does not only depend on the presence of heat release, but also on its position. Oberleithner et al. [22] showed that it is suppressed for V-shape flame types, where the heat release takes place at the upstream part of the recirculation zone, i.e. the gradients of the velocity and density field coincide locally. This is the case for the flames in CTR3 as Fig.18(b) shows. Hence, the actual results agree with these theoretical findings and show that the LES is capable of reproducing the global instability in the isothermal case as well as its damping in the reacting case. 



(a)
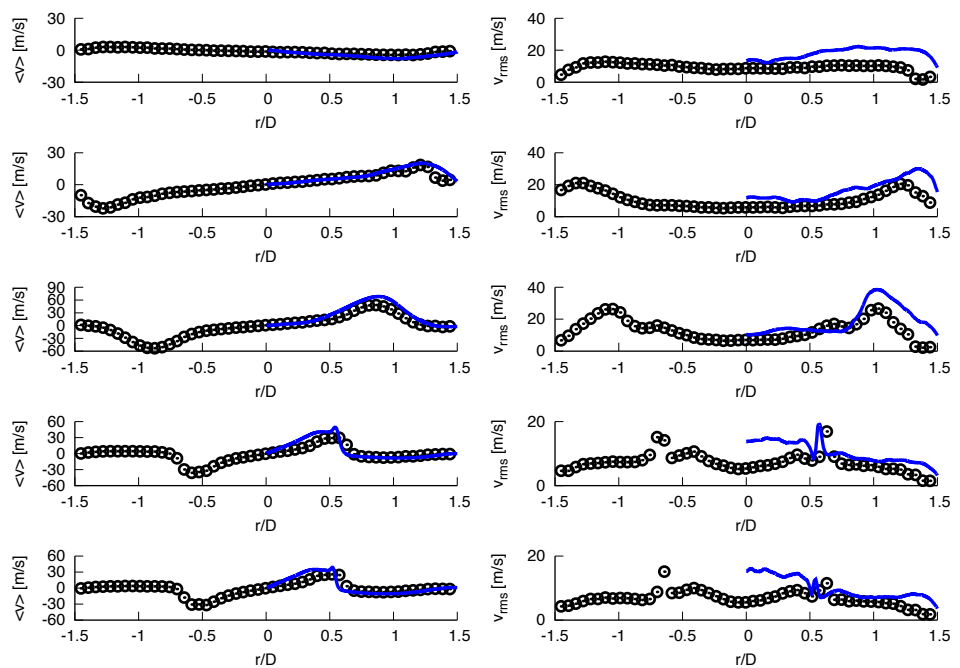

(b)

Fig. 15: Comparison of the numerical simulations with the experimental PIV data for CTR2 at different axial location: (a) mean axial velocity and fluctuations, (b) mean radial velocity and fluctuations. Solid line: LES present work, symbols: experimental data.

In order to further evaluate the influence of the proximity of flashback and the dynamics of the flames under these two conditions, a statistical distribution of the key parameters characterizing the flame evolution is presented using histograms in Fig. 19 The key parameters correspond to the stagnation point at $x=x_{u=0}$, center of the CRZ at $x=x_{u_{\text {min }}}$, flame position $x=x_{f}$ and distance between flame tip and stagnation point $d_{f, 0}=x_{f}-x_{u=0}$. A comparison of the histograms of probabilities obtained for the PIV and LES are shown in Fig.19 (a) and 

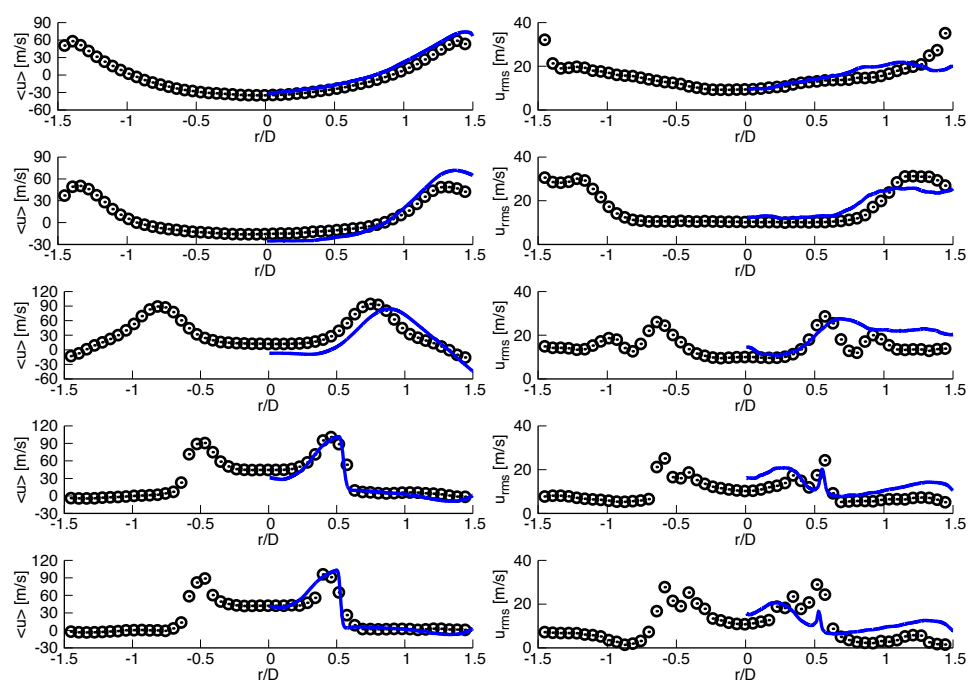

(a)


(b)

Fig. 16: Comparison of the numerical simulations with the experimental PIV data for CTR3 at different axial location: (a) mean axial velocity and fluctuations, (b) mean radial velocity and fluctuations. Solid line: LES present work, symbols: experimental data.

(b). The comparison shows that the numerical simulations were able to reproduce the dynamic behaviour of the stagnation point for the two flames. In general, the LES tends to shift the displacements more upstream than the PIV, and this is magnified as the flame approaches flashback. Nevertheless, the qualitative agreement is satisfactory and the deviations respect the mean value are small in both flames. The same trends are also observed for the dynamics of the CRZ. Both LES and PIV data show a wide probability distribution of the center of the 


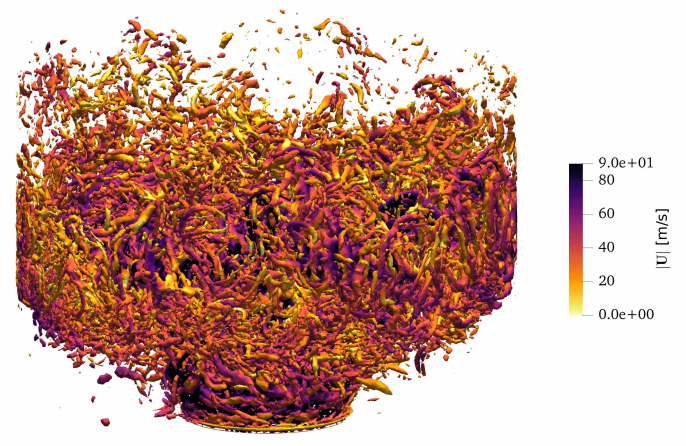

(a)

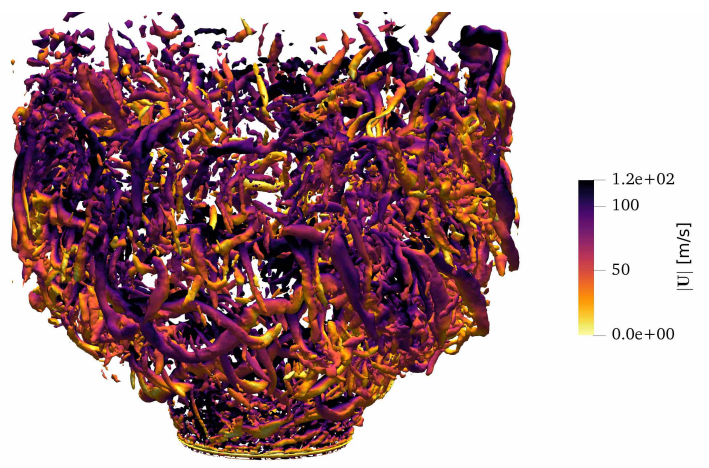

(b)

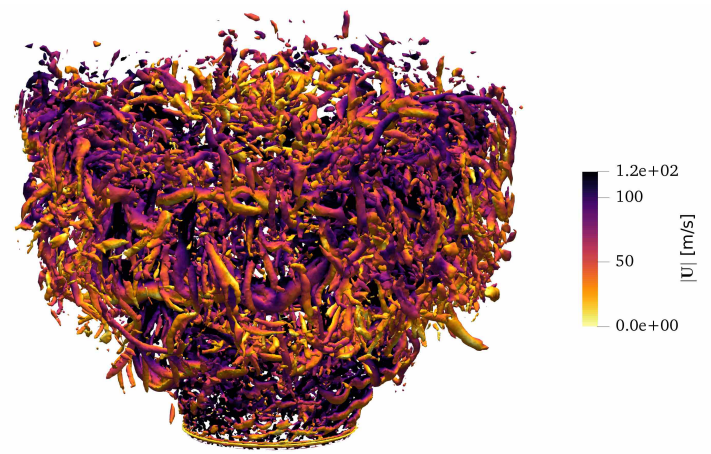

(c)

Fig. 17: Q-vorticity contours coloured by velocity magnitude for: (a) CTR1, (b) CTR2 and (c) CTR3.

recirculation, which shows the transient behaviour of the flow in this region. These results confirm the displacement of the recirculation zone towards the nozzle exit with an increase in flashback propensity as the axial momentum and equivalence ratio are reduced. Probability distributions of the flame position $x_{f}$ and distance $d_{f, 0}$ are shown in Fig. 19 (c) and 




(a)



(b)

Fig. 18: (a) Power spectral density of the absolute velocity fluctuation at an observation point in the combustion chamber $(x=10 \mathrm{~mm}, r=15 \mathrm{~mm})$ of CTR3 in non-reacting (with fuel momentum) and reacting conditions. (b) Density distribution of CTR3 with quivers of the velocity and a contour line of zero axial velocity.

(d) respectively for the LES. The flame in CTR3 is slightly shifted downstream and exhibits higher variations when compared to CTR2, though the evolution is quite similar. The main difference between the cases is observed when computed the relative distance between the flame tip and the stagnation point around the centreline $d_{f, 0}$, see Fig. 19 (d). The dynamics of these structures are rather different. As the flame is operated in the stable range far from the flashback limit (CTR2), there is a well defined separation between the flame and the stagnation point. This distance varies in time with a certain distribution, but it is centered in $20 \mathrm{~mm}$. As the flame approaches flashback (CTR3), the dynamics of this distance change substantially respect to CTR2. The distance in this case is smaller compared to CTR2 up to the point where the most probable locations are around zero suggesting that the flame tip and stagnation point are attached large part of the time. The plot also shows that eventually the flame is decoupled from the stagnation point and this distance also increases up to the level of the CTR2 flame. It is concluded from this analysis that as the flame approaches flashback, the dynamics of the flame are altered specially in this region and the assumption of the perfectly premixed condition might no longer be valid.

\section{Flame structure analysis}

A visualization of the flames using a volumetric rendering of the density gradient is presented in Fig 20 for CTR2 and CTR3 respectively. The flame topology of these swirlstabilized flames can be distinguished in this plot. The flame in CTR2 is characterized by a wider reacting layer with strongest density gradients associated to the higher heat release. It is also observed a relatively higher spreading in the CTR3 flame as it is also found in the velocity fields, see Figs. 13 and 14 




(a)

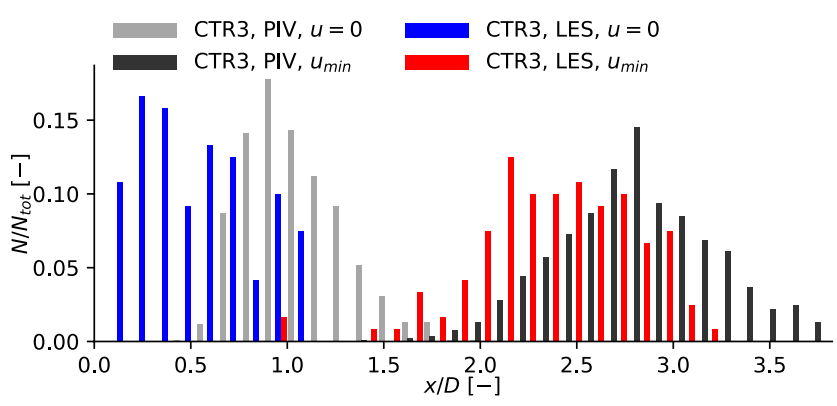

(b)



(c)

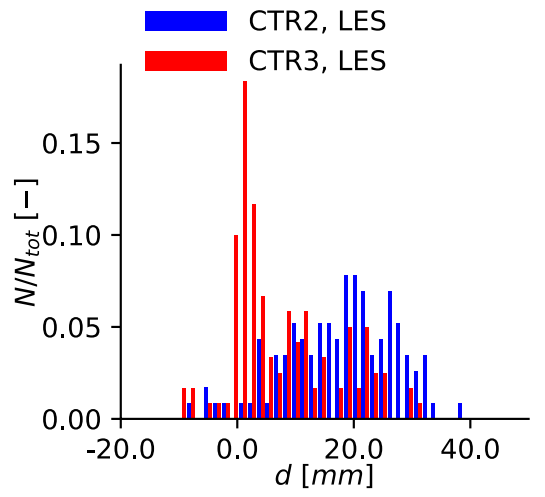

(d)

Fig. 19: Probability distributions of the location of the stagnation point $\left(x=x_{u=0}\right)$ and center of the CRZ $\left(x=x_{u_{\text {min }}}\right)$ for CTR2 (a) and CTR3 (b), flame position $\left(x=x_{f}\right)$ (c) and distance between flame tip and stagnation point $\left(d_{f, 0}=x_{f}-x_{u=0}\right)(\mathrm{d})$.

An analysis of the flame structure is presented in terms of the ability of the proposed modelling approach to capture the radical $\mathrm{OH}$. A sample plot of the distribution of $\mathrm{OH}$ for the investigated flames in stable operation are shown in Fig 21. The comparison indicate 


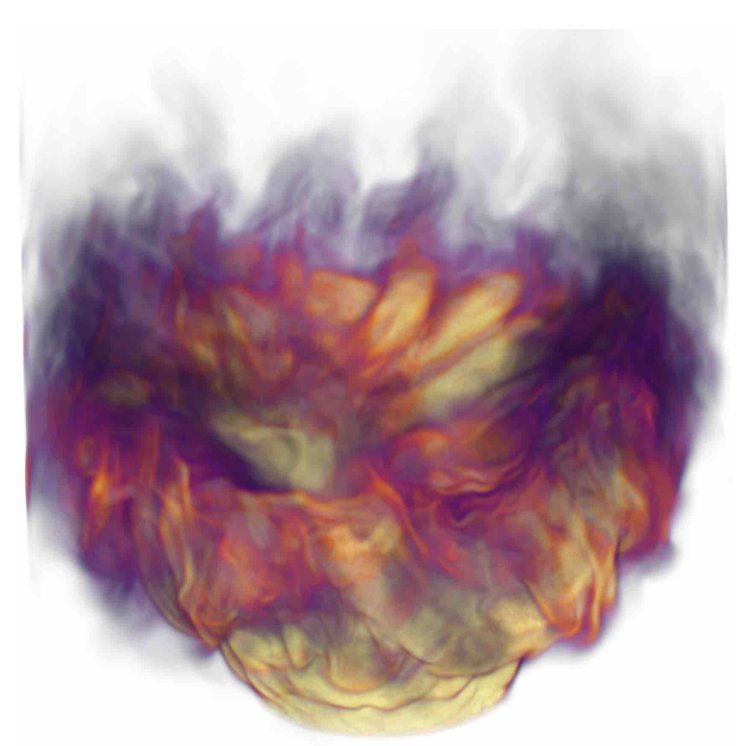

(a)

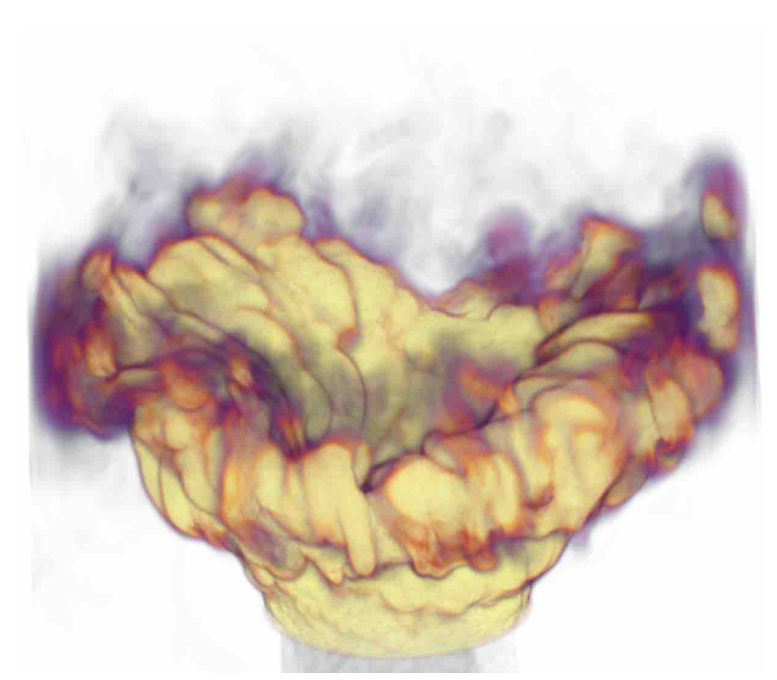

(b)

Fig. 20: Flame visualization using a density gradient for CTR2 (a) and CTR3 (b)

two fundamental sources of disagreement. First, certain discrepancy can be observed in the flame outer layer, where the LES reveals formation of $\mathrm{OH}$ that is not present in the experiment. This over prediction is largely associated to the adiabatic boundary condition imposed in the LES calculation as the outer layer can be influenced by heat to the walls [10, 11. However, the region where the flame stabilizes between the expansion and the central recirculation is the zone with major disagreement. This region is fed by recirculated hot gases with different enthalpies coming from downstream after being cooled from the wall 
along the large CRZ. Despite the CRZ can have a relatively uniform composition due to the long residence time, with sufficient time to mix, the fuel/air mixture is not completely uniform in this region. Besides the lack of disagreement for the $\mathrm{OH}$ formation, Fig. 21 also shows the flame topology is well predicted and the reacting layers predicted by the LES have similar sizes and distributions to those of the OH-PLIF measurements.

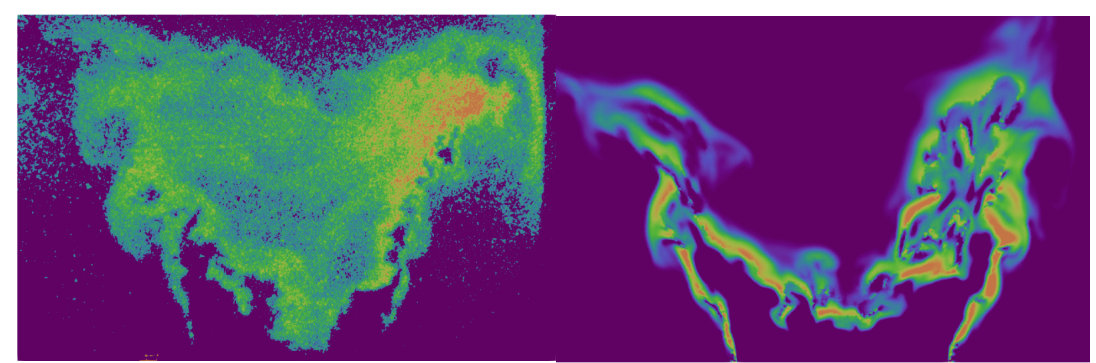

(a)

(b)

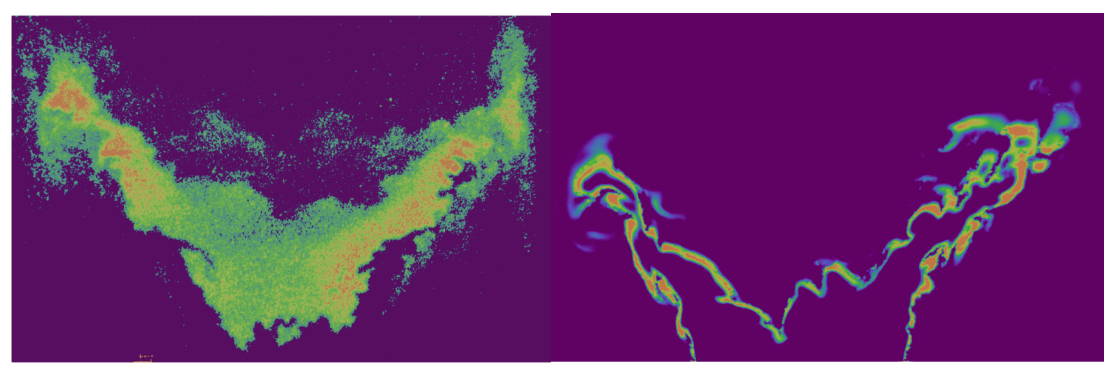

(c)

(d)

Fig. 21: OH distribution for the flames obtained from (a) CTR2: OH-PLIF measurements and (b) CTR2: LES simulation, (c) CTR3: OH-PLIF measurements and (d) CTR3: LES simulation.

It is well known that mixing quality plays an important role in the thermochemical structure of the flames. In this burner, the fuel/air mixture enters relatively homogeneous to the chamber with a slight shift of hydrogen concentration towards the sides of the mixing tube [20]. After the jet expands in the combustion chamber, the mixture results to be slightly leaner on the sides reducing the production of $\mathrm{OH}$ in the outer layer. Additionally, it is also expected that the flame exhibits equivalence ratio fluctuations that can impact the $\mathrm{OH}$ formation. This effect is expected to play a role not only in the overprediction of $\mathrm{OH}$ in the outer layer, but also in the underprediction of $\mathrm{OH}$ in the central part. The results indicate an important reduction of $\mathrm{OH}$ in leaner mixtures even when the flow is assumed to be at equilibrium. Finally, it is expected that radiation will also affect the radical pools and change the chemistry to a certain extend.

In order to better understand what the modelling approach is able to capture in terms of $\mathrm{OH}$ prediction, the distribution of different radicals in progress variable space is given in Fig 22. The plot also includes the distribution of heat release in a shadowed area as an indicator of the iso-surfaces of progress variable having the largest heat release rates. The 
$\mathrm{OH}$ distribution clearly evidences the influence of equivalence ratio in the $\mathrm{OH}$ formation. The $\mathrm{OH}$ produced at $\phi=0.6$ is about twice than the maximum $\mathrm{OH}$ produced at $\phi=0.4$ characterizing CTR3. It is worth noting that also at equilibrium $(c=1)$, the $\mathrm{OH}$ produced for CTR2 is much larger, while there is almost no OH produced in CTR3. The maximum production of $\mathrm{OH}$ occurs near equilibrium and around the same value $(c=0.9)$ for the two flamelets.

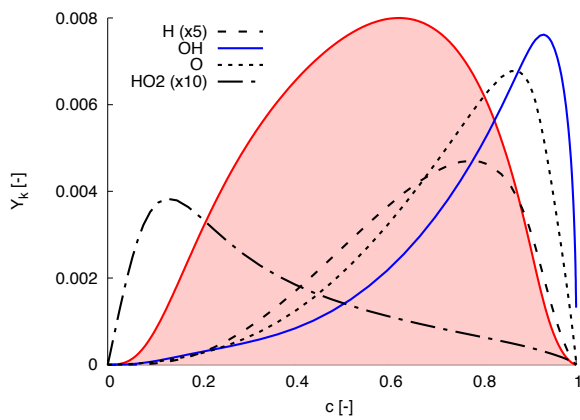

(a)

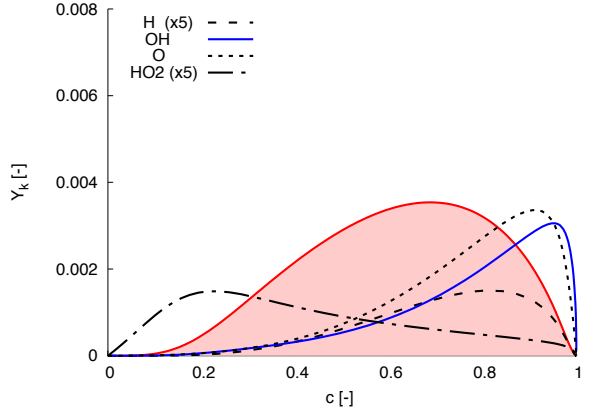

(b)

Fig. 22: Distribution of radicals $\mathrm{H}, \mathrm{OH}, \mathrm{O}$ and $\mathrm{HO}_{2}$ for the flamelets representing CTR2 and CTR3 with the distribution of heat release in shadows along the progress variable space.

Based on the thermochemical structure of unstrained premixed flamelets, the production of $\mathrm{OH}$ by the premixed model is fundamentally given by the flamelet structures in the manifold shown in Fig 22, with the addition of the effect of the subgrid variance that is neglected in this analysis. The numerical results in Fig 21 show $\mathrm{OH}$ levels that correspond mainly to equilibrium, which are low compared to a partially reacted mixture with $c<1$. The presence of large amounts of $\mathrm{OH}$ in the post flame region suggests that this low reactivity mixture could be formed by dilution of the reacting layer with hot gases at lower enthalpy. In fact, the maximum of $\mathrm{OH}$ in the manifold is actually close to equilibrium (see Fig 22), which means that fluctuations in progress variable near equilibrium or equivalence ratio fluctuations will produce larger amounts of $\mathrm{OH}$ compared to the adiabatic perfectly premixed model. This approach was valid to predict hydrodynamic instabilities and flow dynamics, but it is not able to predict with accuracy the $\mathrm{OH}$ formation and the structure of the flames.

\section{Conclusions}

This work presents a numerical study of a technically premixed swirling combustor with central air injection at conditions close to flashback. The burner is designed to operate with high fuel flexibility and flashback resistance, and it is tested here when operated with hydrogen in lean conditions. The stability limits are controlled by the axial momentum imposed in the premixed section that influences the location of the vortex breakdown and the anchoring of the flame. The use of hydrogen-enriched mixtures requires high injection velocities due to the low weight of the fuel. This additional momentum is used in this burner to reduce the 
velocity deficit and control the flashback safety. It was found the flow can go from stable to flashback by reducing the equivalence ratio, which though reduces the axial momentum. This configuration is investigated using low-dissipation numerical schemes and a turbulent combustion model based on presume-shape PDF for turbulence chemistry interactions in the context of large-eddy simulation. The progress variable is defined using the CSP method and scale-resolving simulations are conducted to characterize the flow field under inert and reacting conditions. This study shows the assumption of perfect premixing is valid during the stable operation of the burner up to flashback conditions. The results using a perfectly premixed mixture are well predicted under inert and reacting conditions. The correlation with the experiments is satisfactory for first- and second-order statistics and confirm the validity of the perfect premixing under these conditions. It is found the flow develops a selfexcited oscillation for the inert case in the form of a precessing vortex core. An analysis of the spectra and POD modes indicate that the PVC is attenuated due to the increase in axial momentum and is ultimately suppressed in the reacting flow field. The LES is capable to reproduce both damping effects, which are also known from the experiments. The analysis of the flames have shown certain dynamics as the flashback point is approached. As the axial momentum and equivalence ratio are reduced, the flashback propensity of the burner increases due to an intensification in the velocity deficit of the incoming mixture. Moreover, the recirculation region is shifted upstream, the central recirculation is altered and the flame position is displaced towards the reactants. An analysis of the thermochemical structure of the flames is presented to evaluate the validity of the modelling approach to predict radical formation. OH-PLIF measurements are compared with the $\mathrm{OH}$ predictions by the LES and certain level of disagreement is observed. This modelling approach is found to be valid to predict the hydrodynamic behaviour of the flames in terms of velocity fields and flow oscillations, but it can not predict the $\mathrm{OH}$ formation found in the post combustion zone across the reacting layer. It is discussed that $\mathrm{OH}$ formation is influenced by heat loss and fuel/air mixing and this modelling features should be added to correctly describe the thermochemical structure of the flames.

\section{Acknowledgments}

Funding: The research leading to these results has received funding through the Spanish Ministry of Economy and Competitiveness in the frame of the CHEST project (TRA201789139-C2-2-R). Conflict of Interest: Daniel Mira acknowledges the Juan de la Cierva personal grant IJCI-2015-26686 and Ambrus Both the Marie Skłodowska-Curie grant agreement No. 713673 through the "la Caixa" INPhINIT Fellowship Grant. Computer resources and technical assistance has been provided by the Red Española de Supercomputación (RES) and the Gauss Centre for Supercomputing e.V. (www.gauss-centre.eu) on the GCS Supercomputer SuperMUC at Leibniz Supercomputing Centre (www.lrz.de). The TU Berlin would like to acknowledge the funding received from the EU Seventh Framework Program (FP7/2007-2013) under GA284636 and the European Research Council under the ERC GREENEST with GA247322.

\section{References}

1. Gal Berkooz, Philip Holmes, and John L Lumley. The proper orthogonal decomposition in the analysis of turbulent flows. Annual review of fluid mechanics, 25(1):539-575, 1993. 
2. A. Both, O. Lehmkuhl, and D. Mira. Assessment of low-Mach discretisation strategies for a turbulent channel flow with large density ratios. In International Conference Computational Fluid Dynamics, ICCFD10-323, 2018.

3. S. Burmberger, C. Hirsch, and T. Sattelmayer. Designing a radial swirler vortex breakdown burner. ASME Turbo Expo, pages GT2006-90947, 2006.

4. S. Charnyi, T. Heister, M. A. Olshanskii, and L. G. Rebholz. On conservation laws of navier-stokes galerkin discretizations. J. Comput. Phys., 337:289 - 308, 2017.

5. R. Codina. Pressure stability in fractional step finite element methods for incompressible flows. J. Comput. Phys., 130(1):112-140, 2001.

6. P. Domingo, L. Vervisch, S. Payet, and R. Hauguel. DNS of a premixed turbulent V flame and LES of a ducted flame using a FSD-PDF subgrid scale closure with FPI-tabulated chemistry. Combust. Flame, 143:566-586, 2005.

7. J. Fritz, M. Kröner, and T. Sattelmayer. Flashback in a swirl burner with cylindrical premixing zone. J. Eng. Gas Turbines Power, 126:276-283, 2004.

8. D. Galley, S. Ducruix, F. Lacas, and D. Veynante. Mixing and stabilization study of a partially premixed swirling flame using laser induced fluorescence. Combust. Flame, 158:155-171, 2011.

9. S. Gövert, D. Mira, J.B.W. Kok, M. Vázquez, and G. Houzeaux. Turbulent combustion modeling of a confined premixed methane/air jet flame including heat loss effects using tabulated chemistry. Appl. Energ., 156:804-815, 2015.

10. S. Gövert, D. Mira, J.B.W. Kok, M. Vázquez, and G. Houzeaux. The effect of partial premixing and heat loss on the reacting flow field prediction of a swirl stabilized gas turbine model combustor. Flow Turb. Combust., 100:503-534, 2018.

11. S. Gövert, D. Mira, Miguel Zavala-Aké, J.B.W. Kok, M. Vázquez, and G. Houzeaux. Heat loss prediction of a confined premixed jet flame using a conjugate heat transfer approach. International Journal of Heat and Mass Transfer, 2016. http://dx.doi .org/10.1016/j.ijheatmasstransfer.2016.10.122

12. J.C.R. Hunt, A.A. Wray, and P. Moin. Eddies, stream and convergence zones in turbulent flows. Technical Report Center for Turbulent Research (CTR), (S88), 1988.

13. P. Jochmann, A. Sinigersky, R. Koch, and H.-J. Bauer. URANS prediction of flow instabilities of a novel atomizer combustor configuration. ASME Turbo Expo, pages GT2005-68072, 2005.

14. M. P. Juniper. Absolute and convective instability in gas turbine fuel injectors. ASME Turbo Expo, pages GT2012-68253, 2012.

15. F. Kiesewetter, M. Konle, and T. Sattelmayer. Analysis of combustion induced vortex breakdown driven flame flashback in a premix burner with cylindrical mixing zone. J. Eng. Gas Turbines Power, 129:929936, 2007.

16. O. Lehmkuhl, G. Houzeaux, H. Owen, G. Chrysokentis, and I. Rodriguez. A low-dissipation finite element scheme for scale resolving simulations of turbulent flows. Journal of Computational Physics, in press, 2019.

17. Mechanical and University of California at San Diego Aerospace Engineering (Combustion Research). Chemical-kinetic mechanisms for combustion applications., 2016.

18. D. Mira, X. Jiang, C. Moulinec, and D.R. Emerson. Numerical simulations of turbulent jet flames with non-premixed combustion of hydrogen-enriched fuels. Comput. Fluids, 88:688-701, 2013.

19. D. Mira, X. Jiang, C. Moulinec, and D.R. Emerson. Numerical assessment of subgrid scale models for scalar transport in large-eddy simulations of hydrogen-enriched fuels. Int. J. Hydrogen Energy, 39:71737189, 2014.

20. D. Mira, O. Lehmkuhl, P. Stathopoulos, T. Tanneberger, R. Thoralf, C.O. Paschereit, M. Vazquez, and G. Houzeaux. Numerical investigation of a lean premixed swirl-stabilized hydrogen combustor and conditions close to flashback. In ASME Turbo Expo 2018, GT2018-76229, 2018.

21. K. Oberleithner, M. Stöhr, S.H. Im, C.M. Arndt, and A.M. Steinberg. Formation and flame-induced suppression of the precessing vortex core in a swirl combustor: Experiments and linear stability analysis. Combustion and Flame, 162(8):3100 - 3114, 2015.

22. K. Oberleithner, S. Terhaar, L. Rukes, and C. O. Paschereit. Why nonuniform density suppresses the Precessing Vortex Core. J. Eng. Gas Turbines Power, 135:121506-1, 2013.

23. T. Poinsot and D. Veynante. Theoretical and Numerical Combustion. Ed. Edwards, 3rd Edition, 2012.

24. T.G. Reichel and C. O. Paschereit. Interaction mechanisms of fuel momentum with flashback limits in lean-premixed combustion of hydrogen. Int. J. Hydrogen Energ., 42:4518-4529, 2017.

25. T.G. Reichel, S. Terhaar, and C. O. Paschereit. Increasing flashback resistance in lean premixed swirlstabilized hydrogen combustion by axial air injection. J. Eng. Gas Turbines Power, 137:071503, 2015.

26. S. Roux, G. Lartigue, T. Poinsot, U. Meier, and C. Bérat. Studies of mean and unsteady flow in a swirled combustor using experiments, acoustic analysis, and large eddy simulations. Combust. Flame, 141:40-54, 2011. 
27. V. Seidel, A. Marosky, C. Hirsch, W. Geng, and F. Magni. Influence of the inflow confinement on the flashback limits of a premixed swirl burner. ASME Turbo Expo, pages GT2013-94866, 2013.

28. Y. Sommerer, D. Galley, T. Poinsot, S. Ducruix, F. Lacas, and D. Veynante. Large eddy simulation and experimental study of flashback and blow-off in a lean partially premixed swirled burner. Journal of Turbulence, 5:037, 2004

29. N. Syred. A Review of Oscillation Mechanisms and the Role of the Precessing Vortex Core (PVC) in Swirl Combustion Systems. Progress in Energy and Combustion Science, 32(2):93-161, 2006.

30. Soufien Taamallah, Zachary A. LaBry, Santosh J. Shanbhogue, and Ahmed F. Ghoniem. Thermoacoustic instabilities in lean premixed swirl-stabilized combustion and their link to acoustically coupled and decoupled flame macrostructures. Proc. Combust. Institute, 35(3):3273 - 3282, 2015.

31. E. Tangermann and M. Pfitzner. Evaluation of combustion models for combustion-induced vortex breakdown. Journal of Turbulence, 10:1-21, 2009.

32. T. Tanneberger, T. G. Reichel, O. Krüger, S. Terhaar, and C. O. Paschereit. Numerical investigation of the flow field and mixing in a swirl-stabilized burner with a non-swirling axial jet. In ASME Turbo Expo 2015, GT2015-43382, 2015.

33. Steffen Terhaar, Thoralf G. Reichel, Christina Schrdinger, Lothar Rukes, Christian Oliver Paschereit, and Kilian Oberleithner. Vortex breakdown types and global modes in swirling combustor flows with axial injection. Journal of Propulsion and Power, 31(1):219-229, jan 2015.

34. F. X. Trias and O. Lehmkuhl. A self-adaptive strategy for the time integration of navier-stokes equations. Numerical Heat Transfer, Part B: Fundamentals, 60(2):116-134, 2011.

35. M. Vazquez, G. Houzeaux, S. Koric, A. Artigues, J. Aguado-Sierra, R. Aris, D. Mira, H. Calmet, F. Cucchietti, H. Owen, A. Taha, J. M. Cela, and M. Valero. Multiphysics engineering simulation toward exascale. J. Comput. Sci., 14:15 - 27, 2016.

36. J. Ventosa-Molina, O. Lehmkuhl, C. D. Perez-Segarra, and A. Oliva. Large eddy simulation of a turbulent diffusion flame: Some aspects of subgrid modelling consistency. Flow Turb. Combust., 99:209-238, 2017.

37. A W Vreman. An eddy-viscosity subgrid-scale model for turbulent shear flow: Algebraic theory and applications. Physics of Fluids, 3670(16), 2004.

38. Wei Xiao and Yong Huang. Lean blowout limits of a gas turbine combustor operated with aviation fuel and methane. Heat and Mass Transfer, 52(5):1015-1024, 2016.

39. F. Zhang, P. Habisreuther, M. Hettel, and H. Bockhorn. Modelling of a premixed swirl-stabilized flame using a turbulent flame speed closure model in LES. Flow Turbul. Combust., 82:537-551, 2009

40. H. Zhang and E. Mastorakos. Prediction of global extinction conditions and dynamics in swirling nonpremixed flames using LES/CMC modelling. Flow Turb. Combust., 96:863889, 2016. 